Draft VERSION JunE 22, 2021

Preprint typeset using $\mathrm{LAT}_{\mathrm{E}} \mathrm{X}$ style AASTeX6 v. 1.0

\title{
OBSERVATIONS OF THE CORONAL MASS EJECTION WITH A COMPLEX ACCELERATION PROFILE
}

\author{
A.A. Reva, A.S. Kirichenko, A.S. Ulyanov, and S.V. Kuzin \\ Lebedev Physical Institute, Russian Academy of Sciences, Moscow, Russia; email: reva.antoine@gmail.com
}

\begin{abstract}
We study the coronal mass ejection (CME) with a complex acceleration profile. The event occurred on April 23, 2009. It had an impulsive acceleration phase, an impulsive deceleration phase, and a second impulsive acceleration phase. During its evolution, the CME showed signatures of different acceleration mechanisms: kink instability, prominence drainage, flare reconnection, and a CME-CME collision. The special feature of the observations is the usage of the TESIS EUV telescope. The instrument could image the solar corona in the Fe $171 \AA$ line up to a distance of $2 R_{\odot}$ from the center of the Sun. This allows us to trace the CME up to the LASCO/C2 field of view without losing the CME from sight. The onset of the CME was caused by kink instability. The mass drainage occurred after the kink instability. The mass drainage played only an auxiliary role: it decreased the CME mass, which helped to accelerate the CME. The first impulsive acceleration phase was caused by the flare reconnection. We observed the two ribbon flare and an increase of the soft X-ray flux during the first impulsive acceleration phase. The impulsive deceleration and the second impulsive acceleration phases were caused by the CME-CME collision. The studied event shows that CMEs are complex phenomena that cannot be explained with only one acceleration mechanism. We should seek a combination of different mechanisms that accelerate CMEs at different stages of their evolution.
\end{abstract}

Keywords: Sun: corona-Sun: coronal mass ejections (CMEs)

\section{INTRODUCTION}

Coronal mass ejections (CMEs) are giant eruptions of the coronal plasma into interplanetary space. CMEs occur due to large releases of the magnetic energy on the Sun. Investigations of the mechanisms of the CME acceleration are important for solar physics and questions of the solar-terrestrial connections.

A lot of different models describe CME acceleration (Chen 2011). The onset of the CME could be triggered by MHD instabilities (Török \& Kliem 2005; Kliem \& Török 2006), mass drainage (Fan \& Low 2003; Reeves \& Forbes 2005), or breakout reconnection (Antiochos et al. 1999). The impulsive acceleration of the CME is thought to be connected with the flare reconnection (CSHKP model, Carmichael 1964; Sturrock 1966; Hirayama 1974; Kopp \& Pneuman 1976). CMEs can interact with each other (Lugaz et al. 2017). In the outer corona, the solar wind can accelerate or decelerate the CMEs (Yashiro et al. 2004).

To understand the nature of the CMEs, we need to test these models with experimental data. We need to measure CME acceleration and search for signatures of the acceleration mechanisms.

However, measurements of CME acceleration have difficulties. Today, the early evolution of CMEs is studied with EUV telescopes (for example, AIA, Lemen et al. 2011), and late evolution with the white-light coronagraphs (for example LASCO/C2, C3; Brueckner et al. 1995). The gap between these instruments - altitude range of $1.2-2 R_{\odot}$ - is the "blind zone" for the traditional sets of instruments. At the same time, it is in this altitude range the main $\mathrm{CME}$ acceleration usually occurs.

Observations of the CMEs in the "blind zone" are scarce. They have been performed by the LASCO/C1 coronagraph (Zhang et al. 2001), the Siberian Solar Radio Telescope (Alissandrakis et al. 2013), the Mauna Loa Observatory (Bemporad et al. 2007), the TESIS EUV telescopes (Reva et al. 2014, 2016a,b), the SPIRIT EUV coronagraphs (Slemzin et al. 2008), and the SWAP EUV telescope (Byrne et al. 2014; Mierla et al. 2013; D'Huys et al. 2017). To better understand the $\mathrm{CME}$, we need more CME observations in the altitude range of $1.2-2 R_{\odot}$ from the Sun's center.

In this work, we study the CME that was observed by the TESIS EUV telescope on April 23, 2009. The TESIS EUV telescope could observe the solar corona up to distances of $2 R_{\odot}$ from the Sun's center. The evolution of this event was observed continuously from the solar surface up to the boundaries of the LASCO field of view. 


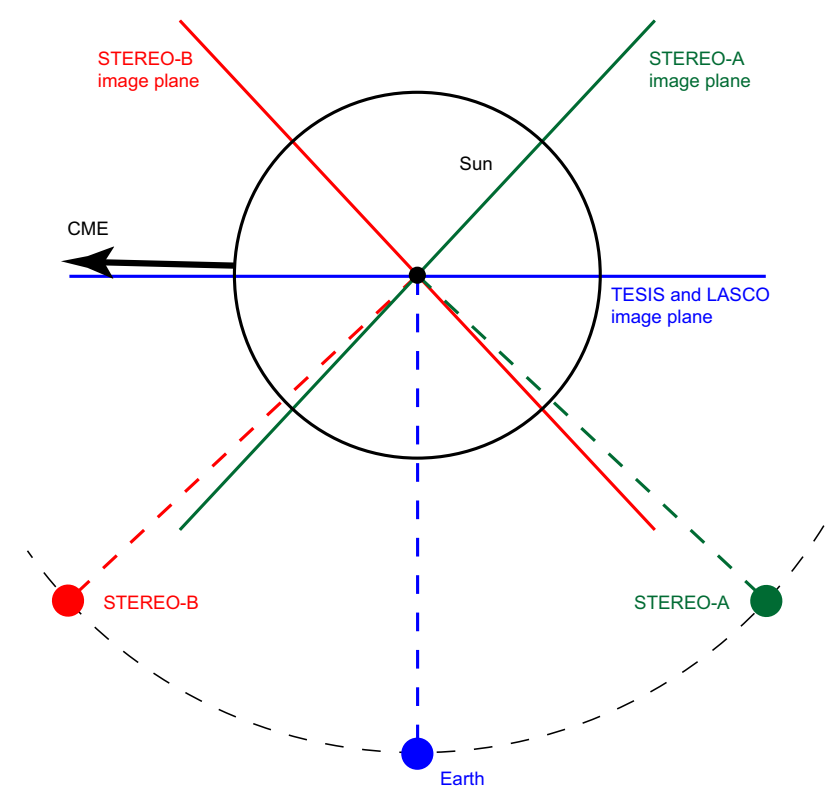

Figure 1. Position of the STEREO satellites.

The studied CME had a complex acceleration profile: two impulsive acceleration phases and one impulsive deceleration phase. The CME showed signatures of several acceleration mechanisms: kink instability, mass drainage, flare reconnection, and a CME-CME collision. The aims of this paper are to present the observations and study the CME acceleration mechanisms.

\section{EXPERIMENTAL DATA}

In this study, we used the data of the TESIS EUV telescopes and the Mg XII spectroheliograph (Kuzin et al. 2011), the SphinX spectrophotometer (Gburek et al. 2011), the LASCO coronagraphs (Brueckner et al. 1995), the EIT telescope (Delaboudinière et al. 1995), and the data of the STEREO satellites (Howard et al. 2008)

TESIS is an instrument assembly that observed the solar corona in the soft X-ray and EUV. It worked on board the CORONAS-PHOTON satellite (Kotov 2011). The TESIS EUV telescope imaged the solar corona in the Fe $171 \AA$ and He $304 \AA$ lines. The special feature of the TESIS EUV telescope was its ability to image the corona up to a distance of $2 R_{\odot}$ from the Sun's center in the Fe $171 \AA$ line (for details, see Reva et al. 2014). We use the TESIS data to observe coronal magnetic structure and prominence evolution at high altitudes.

During the period of the observations, the TESIS $304 \AA$ channel observed the Sun with a 4 min cadence. However, it had data gaps. To fill these data gaps, we used the data of the EIT $304 \AA$ telescope. During the period of the observations, the EIT telescope observed the Sun in the $304 \AA$ line with a 12 min cadence.

The Mg XII spectroheliograph was a part of the TESIS assembly. The instrument built monochromatic images of the solar corona in the Mg XII $8.42 \AA$ line. This line emits at temperatures higher than $4 \mathrm{MK}$, and the $\mathrm{Mg}$ XII images contain only the signal from the hot plasma without any low temperature background. In 2009, the Sun was in the minimum of its activity cycle, and the spectroheligraph Mg XII registered sub-A class flare-like events (Kirichenko \& Bogachev 2017a,b). We use the spectroheliograph Mg XII to study the X-ray emission associated with the analyzed CME.

SphinX is a spectrophotometer that worked on board the CORONAS-PHOTON satellite. SphinX registered solar spectra in the 1-15 keV energy range. In 2009, the solar cycle was in deep minimum, and the GOES flux usually was below the sensitivity threshold. SphinX is more sensitive than GOES, and we use it to see the variation of the $\mathrm{X}$-ray flux.

LASCO is a set of white-light coronagraphs that observe solar corona from $1.1 R_{\odot}$ up to $30 R_{\odot}(\mathrm{C} 1,1.1-$ $3 R_{\odot}$; $\mathrm{C} 2,2-6 R_{\odot}$; C3, 4-30 $\left.R_{\odot}\right)$. In 1998, LASCO C1 stopped working, and today LASCO can only image corona above $2 R_{\odot}$. We use LASCO data to study the CME evolution above $2 R_{\odot}$.

STEREO is a set of two satellites: STEREO-A, which moves ahead of the Earth, and STEREO-B, which moves behind the Earth. During the period of our observations, the STEREO satellites were separated from the Earth by $47^{\circ}$ (see Figure 1). The STEREO satellites carry EUVI telescopes that image the solar corona in the 171, 195, 284, and $304 \AA$ lines. We use the STEREO$B$ EUVI data to determine the $3 \mathrm{D}$ orientation of the erupting prominence and observe the solar surface that is not seen from the Earth point of view.

Moreover, the STEREO satellites carry two whitelight coronagraphs: COR1, which images corona at distances of $1.3-4 R_{\odot}$, and COR2, which images corona at distances of $2-15 R_{\odot}$. We use the COR1 and COR2 data from both satellites to enhance the measurements of the CME kinematics.

We listed the characteristics of the instruments in Table 1 .

\section{RESULTS}

We will present the observations in the following way. Firstly, we will outline the observations. Secondly, we will describe the complete sequence of events. Finally, we will present the analysis performed on the observations.

\subsection{Outline of the Observations}

The CME under study occurred at the north-eastern part of the solar limb on April 23, 2009 (see Figure 2). We recommend the reader to watch the animation to the Figure 2 before proceeding further.

The studied event evolved in the following way. The 
Table 1. Characteristics of the used instruments.

\begin{tabular}{llcrrr}
\hline \hline Satellite & Instrument & Wavelength & Resolution & Field of View & Cadence \\
\hline CORONAS-PHOTON & TESIS EUV telescope & $171 \AA$ & $3.4^{\prime \prime}$ & $\leq 2 R_{\odot}$ & $30-60 \mathrm{~min}$ \\
& & $304 \AA$ & $1.7^{\prime \prime}$ & $\leq 2 R_{\odot}$ & $4 \mathrm{~min}$ \\
& Mg XII spectroheliograph & $8.42 \AA$ & $4.0^{\prime \prime}$ & $\leq 1.6 R_{\odot}$ & $90 \mathrm{~min}$ \\
& SphinX & $1-15 \mathrm{keV}$ & - & - & $1 \mathrm{~min}$ \\
SOHO & $304 \AA$ & $5.3^{\prime \prime}$ & $\leq 1.6 R_{\odot}$ & $12 \mathrm{~min}$ \\
& EIT & white light & $11.4^{\prime \prime}$ & $2-6 R_{\odot}$ & $20 \mathrm{~min}$ \\
LASCO/C2 & white light & $56.0^{\prime \prime}$ & $4-30 R_{\odot}$ & $30 \mathrm{~min}$ \\
LASCO/C3 & $171 \AA$ & $1.6^{\prime \prime}$ & $\leq 1.7 R_{\odot}$ & $2.5 \mathrm{~min}$ \\
& EUVI & $195 \AA$ & $1.6^{\prime \prime}$ & $\leq 1.7 R_{\odot}$ & $10 \mathrm{~min}$ \\
& & $284 \AA$ & $1.6^{\prime \prime}$ & $\leq 1.7 R_{\odot}$ & $20 \mathrm{~min}$ \\
& & $304 \AA$ & $1.6^{\prime \prime}$ & $\leq 1.7 R_{\odot}$ & $10 \mathrm{~min}$ \\
& & white light & $7.0^{\prime \prime}$ & $1.5-4 R_{\odot}$ & $5-10 \mathrm{~min}$ \\
& COR1 & white light & $14.7^{\prime \prime}$ & $2.5-15 R_{\odot}$ & $15 \mathrm{~min}$ \\
\hline
\end{tabular}

prominence started to move up. Later, it twisted in the STEREO-B EUVI images, and a fluxrope appeared in the TESIS Fe $171 \AA$ images.

At the altitude of $220 \mathrm{Mm}\left(0.32 R_{\odot}\right)$ above the solar surface, the prominence tore apart and drained down, while the fluxrope continued to erupt (see Figures 2 and 3). In the LASCO/C2 images, the fluxrope corresponded to the $\mathrm{CME}$ core. By the time the fluxrope had reached the LASCO/C2 field of view, the prominence had already drained down to the solar surface.

At the eastern solar limb, another CME occurred before the studied one. In the $\mathrm{LASCO} / \mathrm{C} 2$ field of view, the studied CME collided with the preceding CME.

The kinematics of the studied CME had an impulsive acceleration phase, an impulsive deceleration phase, and a second impulsive acceleration phase. During the first acceleration phase, we observed signatures of flare reconnection: an increase in the X-ray flux and a two-ribbon flare. There were no signatures of flare reconnection during the second acceleration phase. The second acceleration and the deceleration phases occurred after the CME-CME collision.

The observations are summarized in Figure 4 and Table 2 .

\subsection{Sequence of Events}

The studied CME was associated with a prominence. The TESIS He $304 \AA$ images indicate that the prominence was located at the the north-eastern part of the solar limb. In the STEREO-B EUVI images, the prominence looked like a filament that was inclined by $25^{\circ}$ to the meridian and was close to the TESIS limb (see Figure 5). Therefore, the prominence eruption occurred almost entirely in the TESIS image plane (see Figure 1).

The prominence started to lift at 02:51 UT on April 23, 2009. At 04:28 UT, in the Fe $171 \AA$ images, a
Table 2. Summary of the observations.

\begin{tabular}{ll}
\hline \hline Time, UT & Description \\
\hline$\approx 02: 51$ & prominence started to move up \\
$\approx 04: 27$ & CME core appeared in the TESIS \\
$\approx 04: 35$ & Fe 171 A images \\
$\approx 06: 02$ & prominence twisted \\
$\approx 07: 38$ & prominence started to drain \\
$\approx 07: 38$ & prominence drained down \\
$\approx 07: 00-08: 15$ & the core passed to the LASCO/C2 field \\
$\approx 06: 45-12: 00$ & the SphinX X-ray flux \\
$\approx 07: 00-09: 00$ & the Mg XII spectroheliograph X-ray \\
$\approx 06: 46-07: 20$ & flux \\
$\approx 08: 50$ & motion of the flare ribbons \\
$\approx 08: 40-09: 15$ & impulsive deceleration phase \\
$\approx 09: 15-10: 00$ & the second impulsive acceleration \\
& phase
\end{tabular}

fluxrope formed. In the STEREO-B EUVI images, the prominence twisted at $\approx 04: 35$ UT (see Figure 6 ).

From 06:46 to 07:20 UT, the flare ribbons appeared below the prominence in all four channels of the STEREO-B EUVI (see Figure 7).

We observed an X-source in the Mg XII spectroheliograph images from 07:00 to 09:00 UT (see Figure 8). This source corresponds to the flare arcade below the CME. It was the only X-ray source on the Sun.

During the period of the observations, the GOES $\mathrm{X}$-ray flux was below its sensitivity threshold. From $\approx$ 06:45 UT to 11:00 UT, the SphinX registered an increase of the X-ray flux. Since the Mg XII spectroheliograph observed only one X-ray source, the increase of the SphinX flux comes entirely from the flare arcade. 


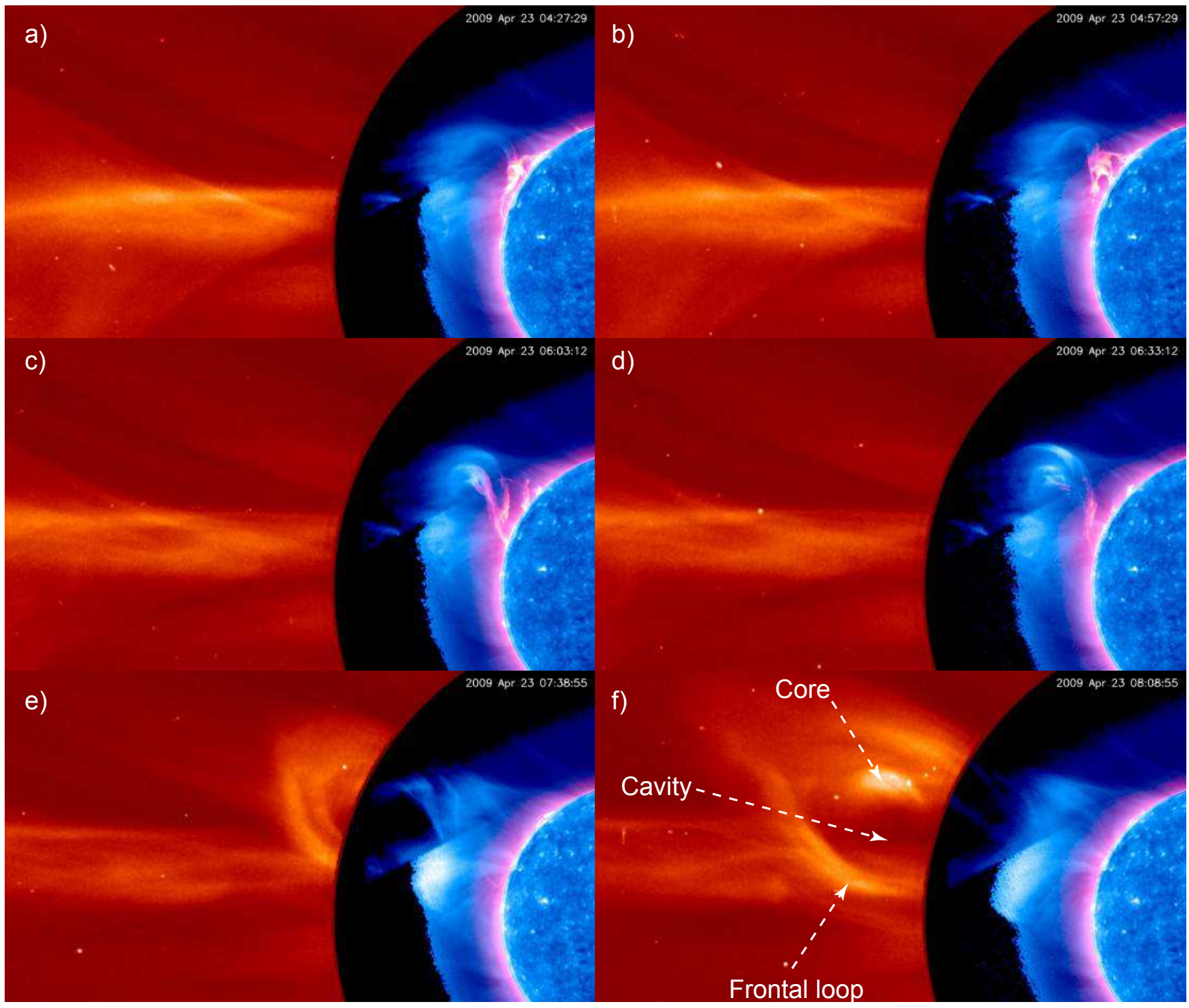

Figure 2. Early evolution of the studied CME. Blue: TESIS Fe $171 \AA$ A images; purple: TESIS He 304 A images; red: LASCO/C2 images. An animation is available for this figure.

We convolved the SphinX spectrum with the response function of the GOES 1-8 A channel and obtained a synthetic GOES flux (see Figure 9, top). The studied flare arcade was of the A0.15 GOES class.

The prominence was located in the lowest parts of the magnetic structure visible in the Fe $171 \AA$ images. While erupting, the magnetic structure dragged the prominence up. At the altitude of $220 \mathrm{Mm}\left(0.32 R_{\odot}\right)$ above the solar surface, the prominence tore apart and started to drain while the fluxrope continued to erupt (see Figures 2 and 3 ).

The gap between the LASCO/C2 and TESIS fields of view is small. This allows us to track the CME without losing it from sight. In the $\mathrm{LASCO} / \mathrm{C} 2 \mathrm{im}-$ ages, the CME had a three-part structure: core, cavity, and frontal loop (see Figure 2f). The CME core in the
LASCO images corresponds to the fluxrope center observed in the Fe $171 \AA$ images. When the CME core left the TESIS field of view, the prominence had already drained down.

At the eastern solar limb, another CME occurred before the studied one. In the LASCO/C2 field of view, the studied CME collided with the preceding CME at $\approx 08: 50$ UT (see Figure 10).

\subsection{Analysis}

\subsubsection{CME Kinematics}

We measured the coordinates of the CME core (its central part) and the CME frontal loop (its farthest part) in the TESIS Fe $171 \AA$ and He $304 \AA$, LASCO $\mathrm{C} 2$ and $\mathrm{C} 3$ images (see Figure 10). We used a simple point and click method. The procedure was repeated 


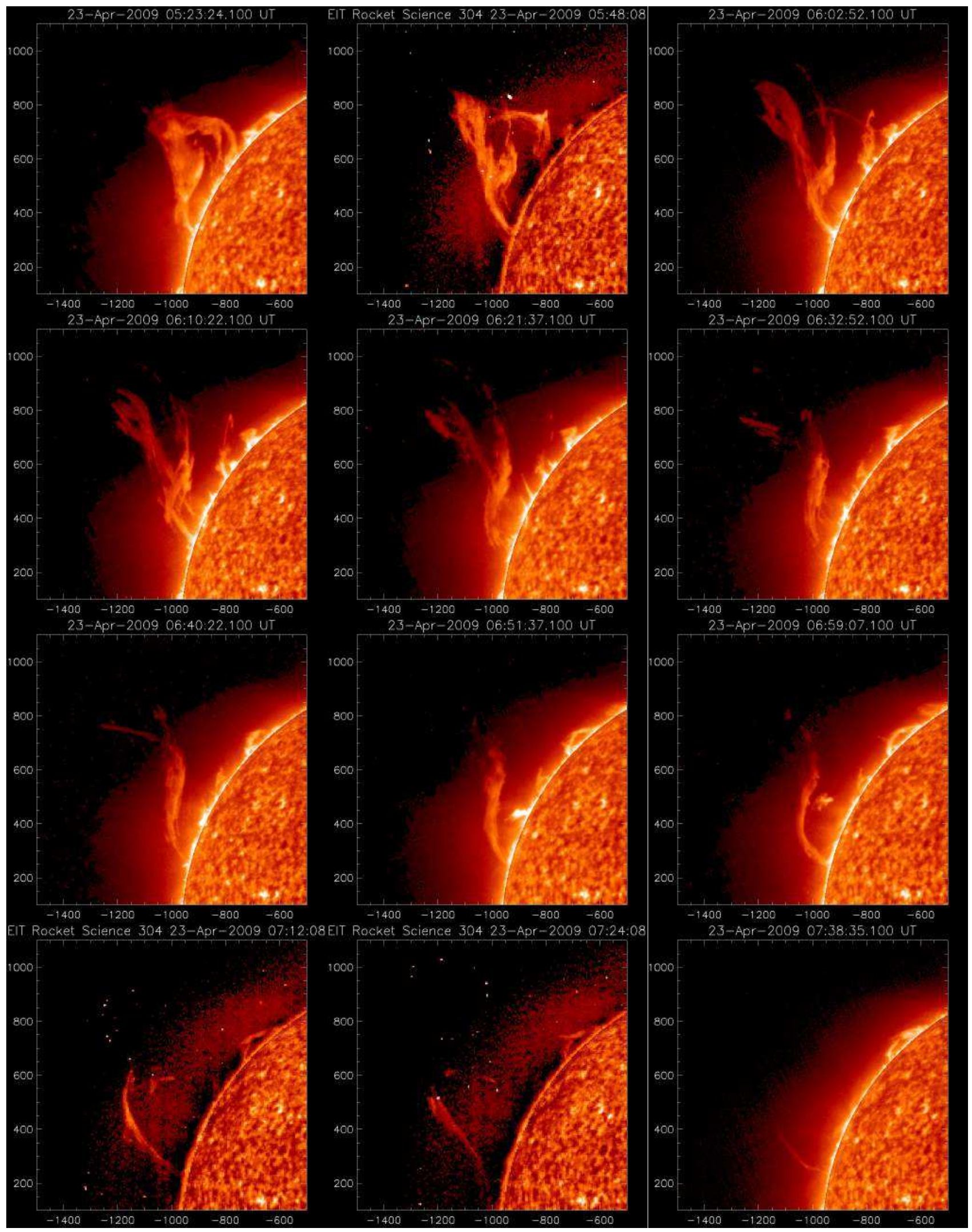

Figure 3. Draining of the prominence. These are TESIS and EIT He $304 \AA$ images. To increase the prominence's visibility, we lowered the disk intensity (multiplied by 0.2). Coordinates are measured in arcseconds. An animation is available for this figure. 


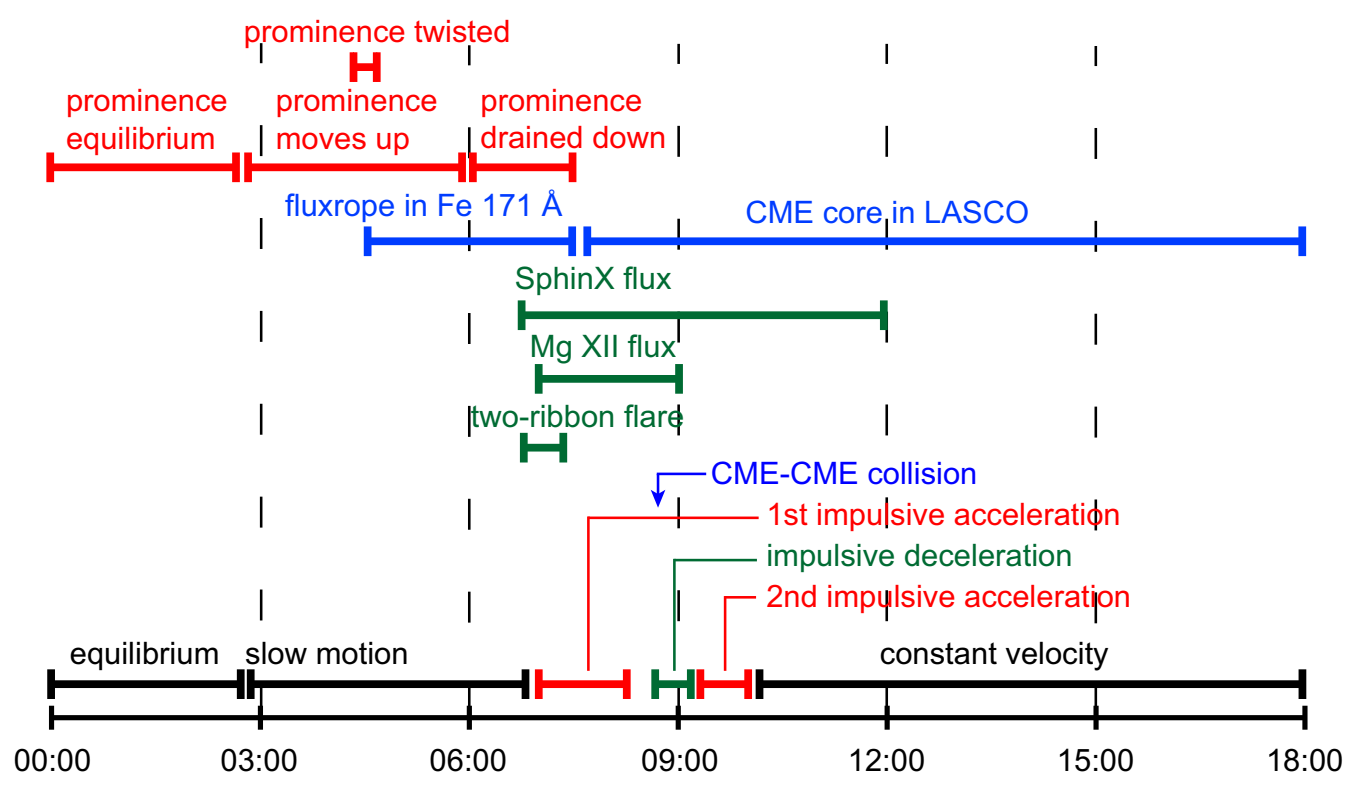

Figure 4. Summary of the observations.

nine times to make this method less subjective, and to estimate error bars. Using the obtained coordinates, we calculated the distance from the Sun's center $(r(t))$.

The studied CME was also observed by the STEREO$A$ (EUVI $195 \AA$, COR1 and COR2) and STEREO-B (COR1 and COR2). We measured the CME core and frontal loop trajectories in these images using the same method.

We want to combine the kinematics measured with TESIS, LASCO, and STEREO. However, TESIS/LASCO, STEREO-A, and STEREO-B observed the event from different view-points. The instruments responded differently to temperature and density. The positions of the CME core and frontal loop were determined subjectively. Due to these factors, if we put the values $r(t)$ measured by different instruments on the same plot, the values will slightly differ from each other (even after the correction of the projection effects).

To correct the discrepancy, we adopted the procedure from Reva et al. (2016b). First, we scaled the values obtained from STEREO-A and STEREO-B using the separation angles of the satellites. After this procedure, the STEREO points slightly deviated from the TESIS/LASCO points.

We assumed that the CME expanded uniformly, and scaled each of the STEREO channels to fit the TESIS/LASCO plot (see Table 3). As a result, we obtained a composite kinematics plot consisting of 139 points for the $\mathrm{CME}$ core and 61 points for the frontal loop (see Figures 11, 12, and 13, top).

We want to numerically differentiate $r(t)$ and obtain radial velocity $v(t)$ and radial acceleration $a(t)$. Since
Table 3. Kinematics scaling multiplier.

\begin{tabular}{lll}
\hline \hline Channel & Core & Frontal loop \\
\hline STEREO-A EUVI 195 & 0.93 & - \\
STEREO-A COR1 & 0.83 & 0.82 \\
STEREO-A COR2 & 1.00 & 0.80 \\
STEREO-B COR1 & 0.82 & 0.90 \\
STEREO-B COR2 & 0.97 & 0.84 \\
\hline
\end{tabular}

the studied CME collided with another one, we expect its acceleration profile to be complex. Therefore, we should use a numerical differentiation method that does not make any assumptions about the shape of the acceleration profile.

Numerical differentiation of the experimental data is a complex task, because the measurements are prone to errors. Application of the straightforward finite difference method will lead to a highly noisy result. To tackle this problem, a lot of numerical differentiation techniques were developed (for a review, see Wood 1982).

To find derivatives, we chose the local least-square approximation method (see pages 320-324 of Wood 1982). For each point, we fitted the plot with a line using only data from the small vicinity of the point. The tangent of the slope of the fitted line is the derivative.

The size of the vicinity affects the result. If the vicinity is too large, the derivative will be smooth, but the fastchanging details of the derivative will be lost. If the vicinity is too small, we will see the fast dynamics of the derivative, but the derivative will be too noisy.

In our research, we varied the size of the vicinity along the plot. We wanted to make the size of the vicinity as small as possible, and, at the same time, keep the 


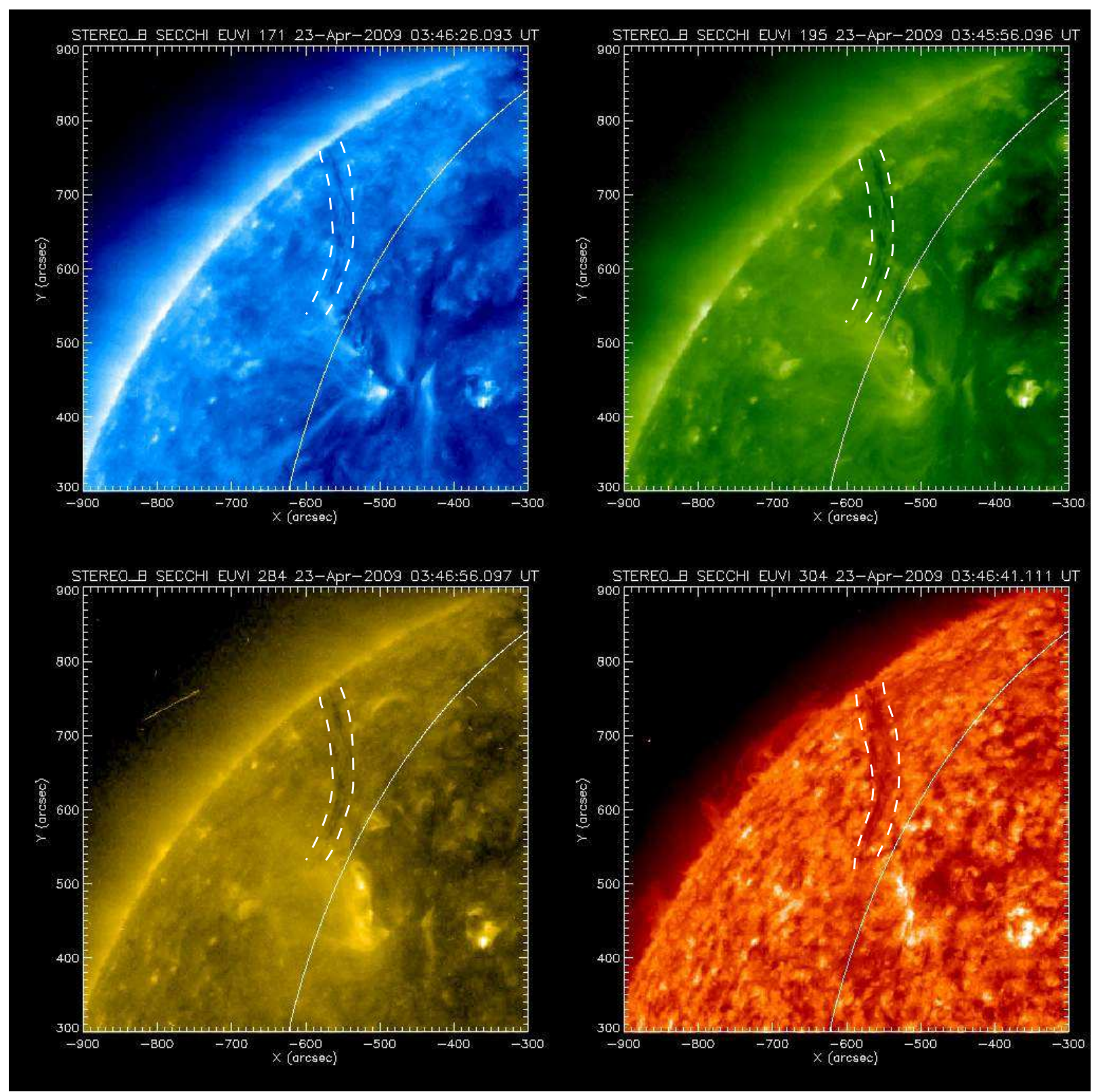

Figure 5. Erupting prominence (filament) in the STEREO EUVI-B images. Dashed lines mark the filament; the solid line designates the TESIS limb.

derivative smooth.

The result is presented in Figures 11, 12, and 13. The kinematics of the CME core and frontal loop look similar.

1. Before $\approx$ 07:00 UT, the CME slowly accelerated.

2. From $\approx 07: 00$ to $\approx 08: 15 \mathrm{UT}$, the CME impulsively accelerated.

3. From $\approx 08: 15$ to $\approx 08: 40 \mathrm{UT}$, the CME moved with a constant velocity.

4. From $\approx 08: 40$ to $\approx 09: 15 \mathrm{UT}$, the CME impulsively decelerated.
5. From $\approx 09: 15$ to $\approx 10: 00 \mathrm{UT}$, the CME impulsively accelerated.

6. After $\approx 10: 00 \mathrm{UT}$, the CME moved with approximately constant velocity.

\subsubsection{Flare Ribbons Motion}

During the eruption, a two-ribbon flare occurred below the CME (see Figure 7). The speed of the ribbon separation reflects the reconnection rate in the current sheet (Qiu et al. 2004). The faster the ribbons move, the higher the reconnection rate.

We measured the distance between the ribbons in the EUVI-B images using a point-and-click procedure. The 


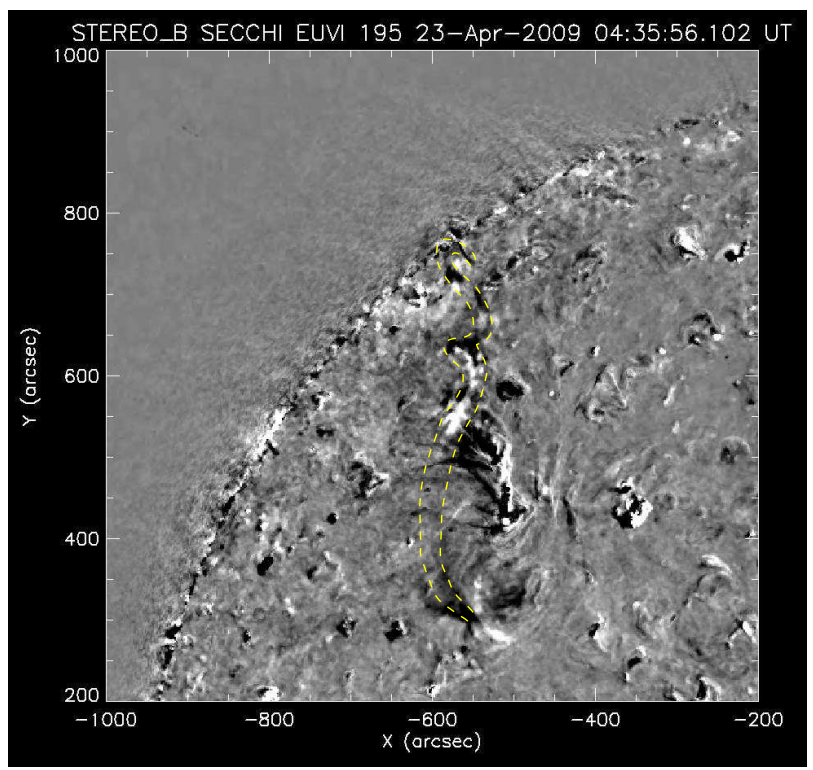

Figure 6. Twisting of the prominence. This is a running difference image from the EUVI-B $195 \AA$ channel. The yellow dashed lines indicate the prominence.

main source of errors in this method is the subjectivity. To eliminate subjectivity and estimate error bars, we repeated the procedure multiple times. Then we corrected the result for the projection effect.

Before 06:46 UT, the two-ribbon flare was not seen in the EUVI-B images. We added "zero points" to the plot: zero values at times when the EUVI-B took images, but the two-ribbon flare was absent.

Using local least-square approximation method, we numerically differentiated the measured distances and obtained the ribbons' separation velocity. From 06:45 UT to 07:15 UT, the ribbons moved with a roughly constant speed of $15 \mathrm{~km} \mathrm{~s}^{-1}$ (see Figure 14). After 07:15 UT, the distance between the footpoints was constant within error margins.

\subsubsection{X-ray Flux}

The derivative of the soft X-ray flux reflects the reconnection rate (Neupert 1968). Using the local leastsquare approximation method, we numerically differentiated the SphinX X-ray flux.

The derivative of the SphinX flux and the flare ribbons velocity have peaks that correlate with the first impulsive CME acceleration phase (see Figure 9, bottom). During the second acceleration phase, there were no peaks of the SphinX X-ray flux or ribbon velocity.

\section{DISCUSSION}

\subsection{Initiation of the $C M E$}

In the early stages, the prominence associated with the CME twisted (see Figure 6). We think that this is a manifestation of helical kink instability (Hood \& Priest
1981). Later, the prominence drained down. Below, we will discuss the role of these two mechanisms in the triggering of the observed CME.

The kink instability could lead to a CME or a failed eruption. Török \& Kliem (2005) showed that, if the magnetic field falls off rapidly in the vicinity of the fluxrope, then kink instability will lead to the eruption. Otherwise, a failed eruption will happen.

In the mass-unload model, a CME erupts due to the draining of mass from the CME pre-erupting structure (Fan \& Low 2003). If the magnetic field is strong, the effect of gravity is negligible. If the magnetic field is weak, the presence of mass will make the fluxrope more stable (Reeves \& Forbes 2005). If, for some reason, part of the CME mass drains out, the equilibrium could be lost, and the CME will erupt.

Our observations differ from the mass-unload model. In the mass-unload model and observations that support it (Seaton et al. 2011), the CME loses mass before an eruption. In our observations, the CME lost the mass after the onset of the eruption.

Aulanier (2014) analyzed existing CME models and came to the conclusion that only two physical mechanisms can drive the onset of a CME: torus instability (Kliem \& Török 2006) and breakout reconnection (Antiochos et al. 1999). Other mechanisms could facilitate an eruption or bring the system to the point where torus instability or breakout reconnection will occur.

We see two possible ways to interpret the observations. The first one is that, after experiencing kink instability, the fluxrope reached an altitude where the magnetic field fell off rapidly enough for the torus instability to occur. In this scenario, the torus instability drove the CME onset. The draining played only an auxiliary role: it decreased the CME mass, which helped to increase the CME acceleration.

The second option is that, after the kink instability, the fluxrope reached a meta-stable state. In this case, the draining caused CME onset.

However, we observe that the prominence was continuously rising without reaching any meta-stable state. Therefore, we think that the first interpretation explains the observations more correctly than the second one.

\subsection{Two Impulsive Acceleration Phases}

The studied CME had two impulsive acceleration phases. A similar effect was reported by Su et al. (2012) and Byrne et al. (2014). The authors analyzed the CME with the two-stage acceleration (both works studied the same event). In their works, each stage of the acceleration was accompanied by the X-ray flux peak. They interpreted the second X-ray peak as a second stage of magnetic reconnection, which caused the second acceleration phase. 


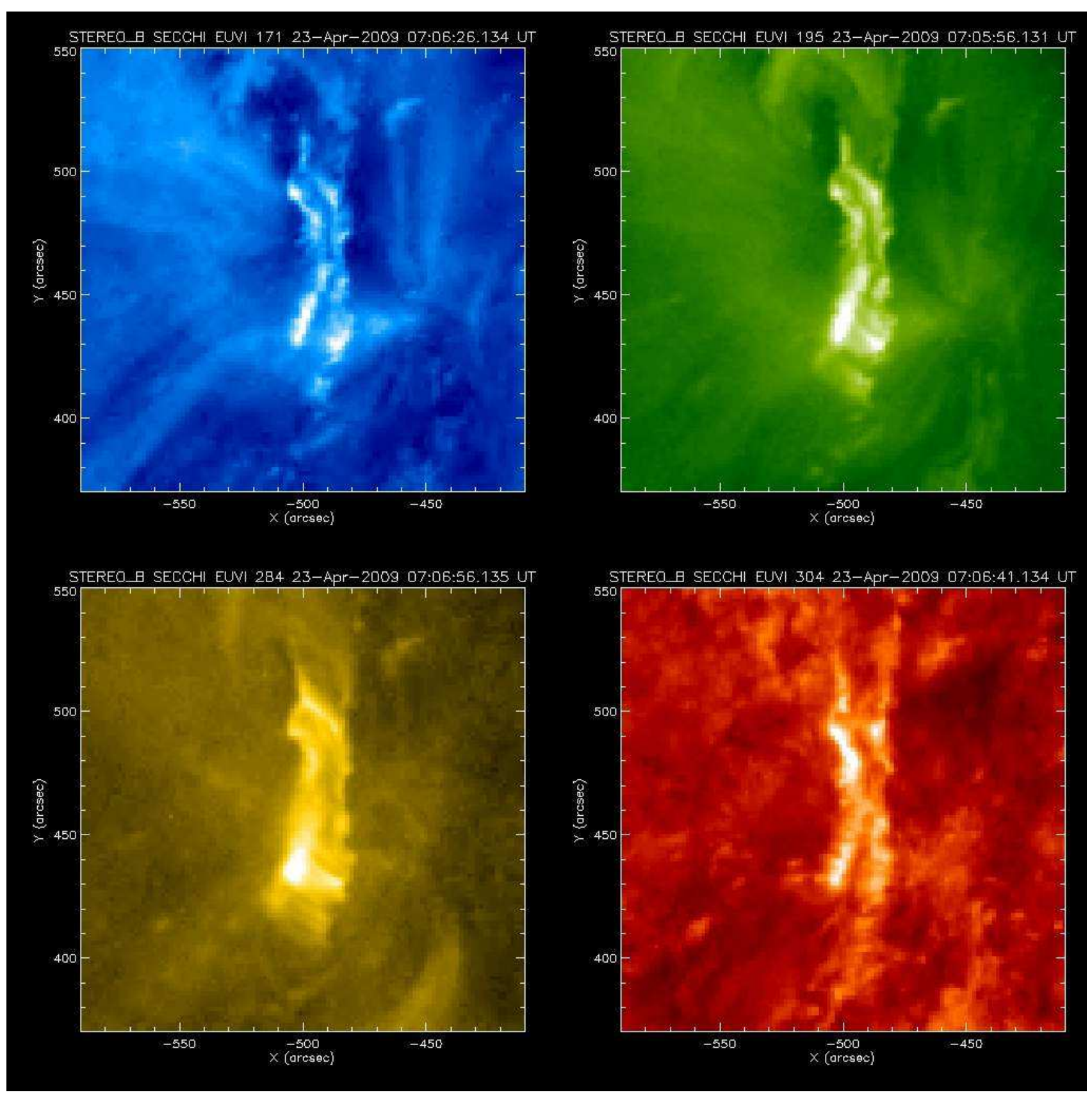

Figure 7. Two-ribbon flare observed by STEREO-B EUVI.

Gosain et al. (2016) reported another CME with a two-stage acceleration. The authors calculated the decay index of the coronal magnetic field and found that there were two altitude ranges for the prominence stability. They concluded that the two areas of stability caused a two-stage acceleration of the CME.

The above-mentioned papers reported two-stage acceleration low in the corona. In this work, the second impulsive acceleration phase occurred at a height of several solar radii. This is a notable observation that deserves discussion.

During the first impulsive acceleration phase, the two- ribbon flare occurred. The speed of the ribbon separation and the derivative of the X-ray flux (signatures of the flare reconnection) peaked at the beginning of the first impulsive acceleration phase (see Figure 9). We think that the first acceleration phase was caused by the flare reconnection described by the standard CME model (CSHKP model, Carmichael 1964; Sturrock 1966; Hirayama 1974; Kopp \& Pneuman 1976).

We believe that the temporal correlation between the first acceleration phase and signatures of the flare reconnection is not a coincidence. For the majority of CMEs, the impulsive acceleration is correlated with the 


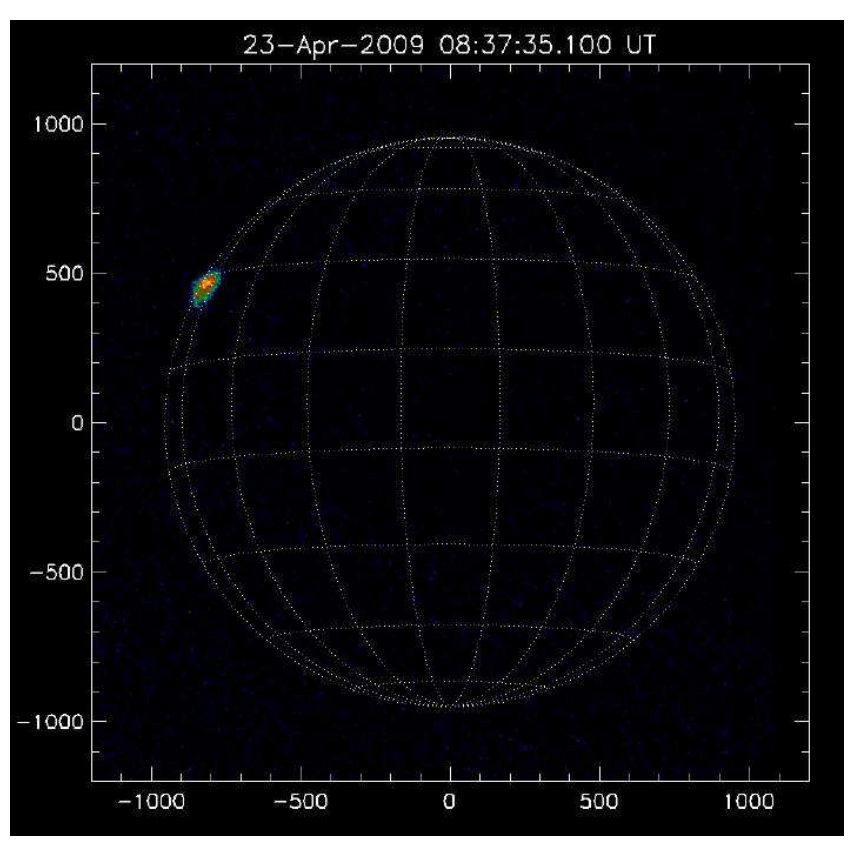

Figure 8. Hot plasma observed below the studied CME with the Mg XII spectroheliograph. Yellow and red correspond to high intensities, while blue and green correspond to low intensities. Coordinates are measured in arc seconds.

rise of the soft X-ray flux (Maričić et al. 2007; Bein et al. 2012), the hard X-ray bursts (Temmer et al. 2008, 2010), and peak of the reconnection rate (Qiu et al. 2004).

There are no cause-effect relationship between flares and CMEs. Both phenomena result from the same complex magnetic process (Webb \& Howard 2012; Chen 2011). The flare reconnection increase the CME acceleration, and faster motion of the CME increase the reconnection rate (Schmieder et al. 2015).

The second impulsive acceleration phase was preceded by the deceleration phase. Right before the deceleration phase, the studied CME collided with another CME. We think that both the deceleration and acceleration were caused by the complex dynamics of the CME-CME interaction: deceleration corresponds to CME compression, while acceleration corresponds to CME restitution. Shen et al. (2012b) performed MHD simulation of the CME-CME collision and showed that, during the collision, the CME experiences both acceleration and deceleration.

It may look strange that the studied CME increased its velocity after the collision. However, the CME-CME collision is a complex interaction of two magnetic plasma clouds that occurs in 3D. Furthermore, CME-CME collision could be super-elastic (Shen et al. 2012a, 2013). Therefore, it is possible that the studied CME could increase its speed as a result of the collision.

The CME-CME collisions observed in the heliosphere lasted for 8-24 hours. This duration roughly coincides with the Alfven crossing time of a CME (Lugaz et al. 2017).

We estimate the CME-CME collision time $(\tau)$ as a total duration of the impulsive deceleration and the second impulsive acceleration phases $(\tau \approx 100 \mathrm{~min})$. The corresponding Alfven speed $\left(v_{A}\right)$ should be equal:

$$
v_{A} \approx \frac{L}{\tau} \approx 250 \mathrm{~km} \mathrm{~s}^{-1},
$$

where $L$ is the size of the studied CME during the collision $\left(L \approx 2 R_{\odot} \approx 1500 \mathrm{Mm}\right)$. The value of $v_{a}$ coincides within the order of magnitude with the Alfven speed inside CMEs.

During the CME collision, the acceleration profiles of the CME core and frontal loop look similar: they both initially impulsively decelerate and then impulsively accelerate. However, the values of the acceleration differ. This means that different parts of the CME react differently to the collision.

\subsection{CME Core and a Prominence}

In white-light coronagraph images, approximately $30 \%$ of CMEs have a three-part structure: a bright core, a dark cavity, and a bright frontal loop (Illing \& Hundhausen 1985; Webb \& Hundhausen 1987). It is possible that more CMEs have a three-part structure, but we do not see it due to projection effects.

Illing \& Athay (1986) compared white-light and $H_{\alpha}$ images of the $S M M$ coronagraphs (Hundhausen 1999), and showed that several CMEs contained cold prominence plasma inside their cores. In white-light coronagraphs images, the CME core often looks like a prominence (a long linear twisted structure; Plunkett et al. 2000). Since the core resembles the prominence and some CME cores contained cool prominence plasma, the core is usually interpreted as a cool erupting prominence (House et al. 1981; Webb \& Howard 2012; Parenti 2014).

However, a CME core could have a wide range of temperatures: cool (0.03-0.3 MK; Akmal et al. 2001; Ciaravella et al. 1997, 1999, 2000), warm $(\approx 1$ MK; Ciaravella et al. 2003; Landi et al. 2010; Reva et al. 2016a), or hot (5-10 MK; Reeves \& Golub 2011; Song et al. 2014; Nindos et al. 2015). The warm and hot plasma inside the CME cores could be interpreted in two ways: the prominence is heated during the eruption (Filippov \& Koutchmy 2002) or the CME core is not a prominence. As we see, the relationship between the core and the prominence is unclear.

In this work, we studied a CME with a three-part structure in the LASCO images. Its core corresponded to the fluxrope, as observed in the Fe $171 \AA$ images. However, TESIS He $304 \AA$ images showed that the prominence drained down, before the CME reached the 

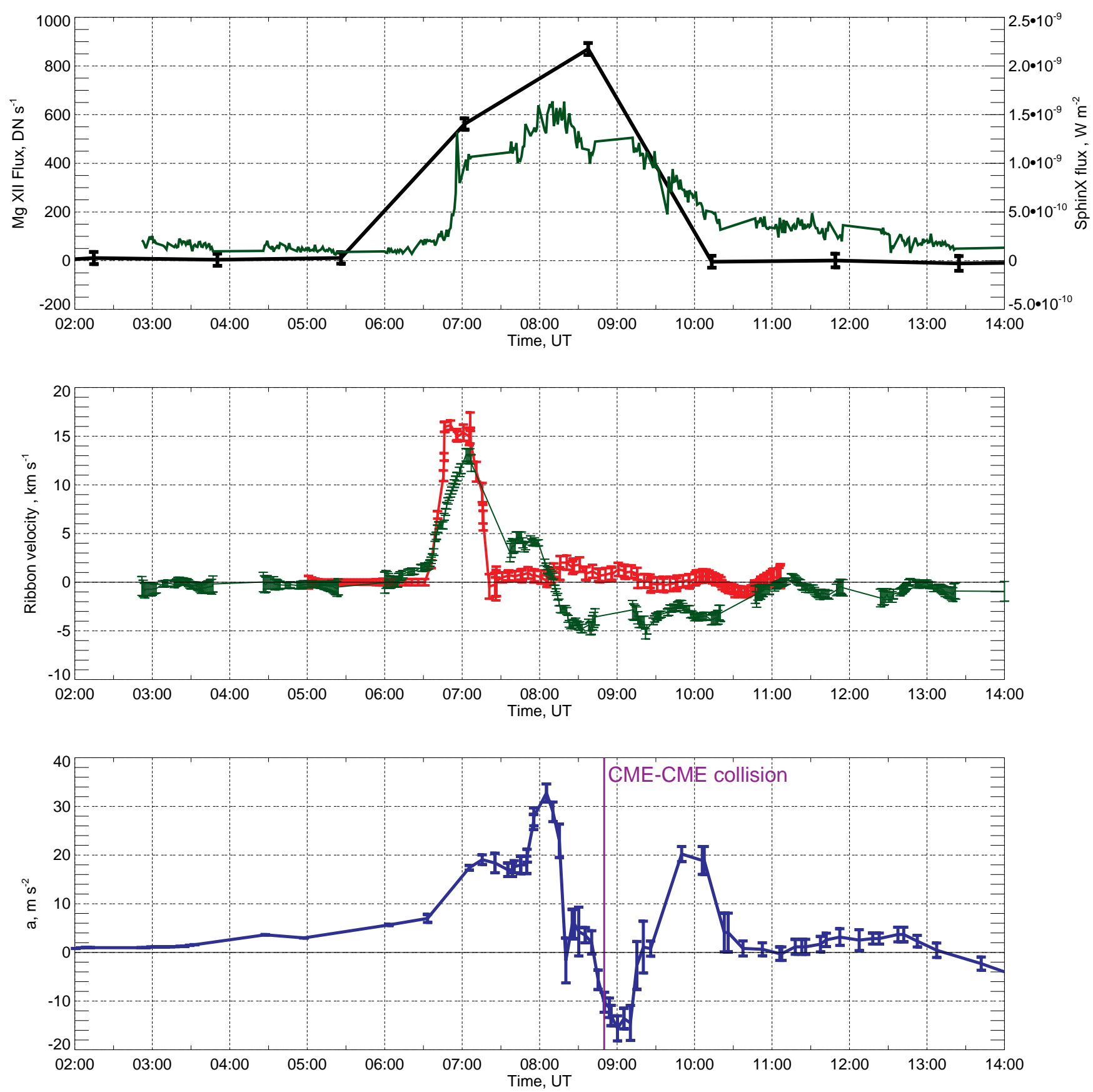

Figure 9. Comparison of the X-ray flux, the acceleration of the CME core, and the separation velocity of the flare ribbons. Black: the flux of the hot source observed with the Mg XII spectroheliograph; green top: the SphinX flux convolved with the response function of the GOES 1-8 A channel; green middle: the derivative of the SphinX flux; red: the separation velocity of the flare ribbons; blue: the acceleration of the CME core. Purple line marks the time of the CME-CME collision.

\section{LASCO field of view.}

The apparent drainage of the prominence could be explained in several ways. Firstly, the prominence could actually drain down. In this case, the core would not contain the prominence plasma. Probably, the core observed in LASCO images corresponded to the warm $(\approx 1 \mathrm{MK})$ fluxrope that surrounded the prominence.

Secondly, it is possible that the small part of the prominence escaped with the CME, but we did not observe this, due to the limited cadence or sensitivity of the instruments. In this case, the core would correspond to the escaped part of the prominence.

Finally, the prominence could be heated during the eruption in such a way that it creates the illusion of drainage. In this case, the core would contain not a cool prominence plasma, but rather a heated promi- 


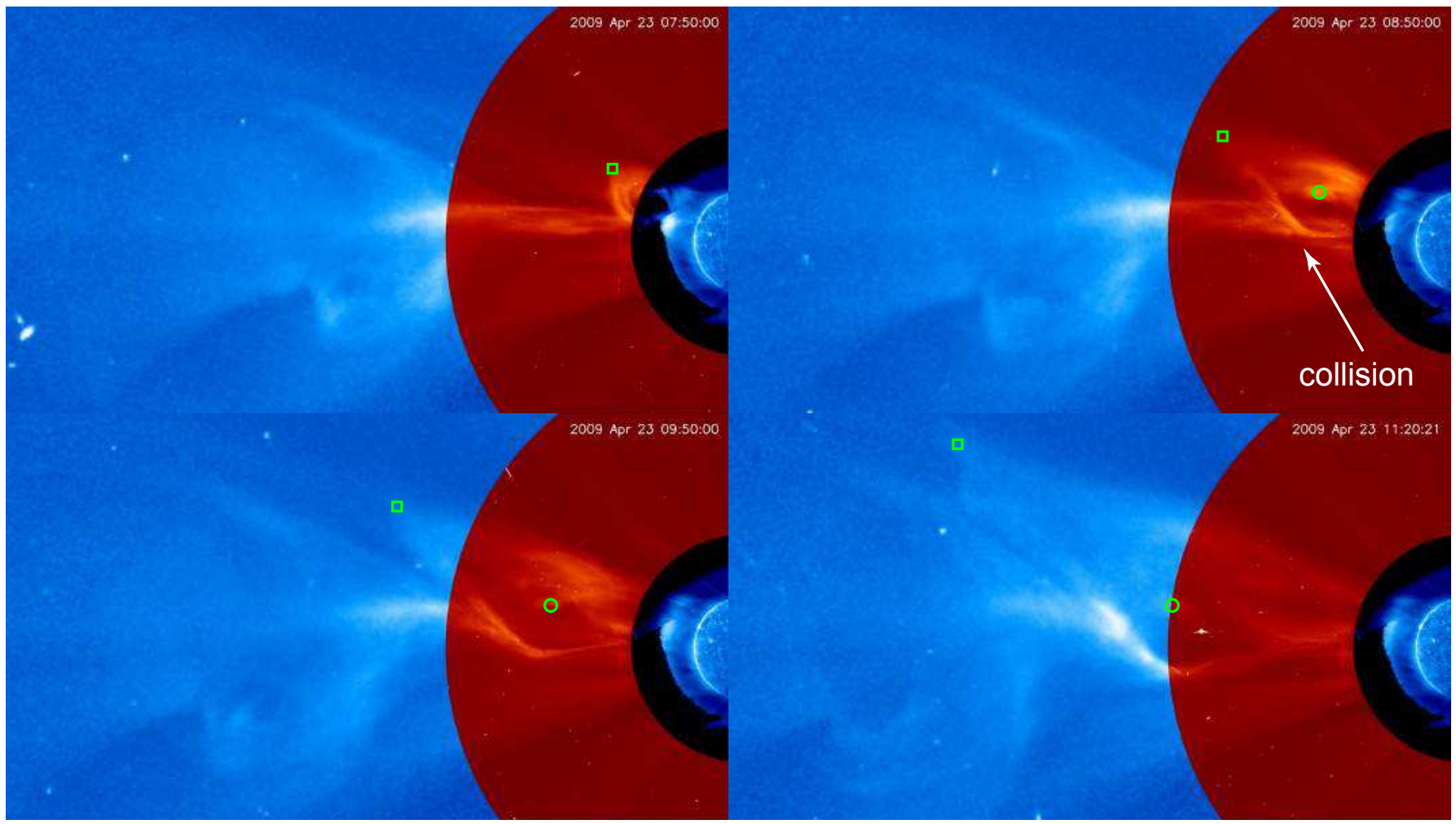

Figure 10. CME-CME collision. Outer blue: LASCO/C3 images, red: LASCO/C2 images, inner blue: TESIS Fe $171 \AA$ images. Circle marks the CME core. Rectangle marks part of the frontal loop. Marked parts were used to measure CME kinematics. An animation is available for this figure.

nence plasma.

Although the CME core in the white-light images is often interpreted as a cold erupting prominence, our observations suggest that this assumption is not always correct. Evolution of the erupting prominence in the distance range of $1.2-2 R_{\odot}$ requires further investigation.

\section{CONCLUSION}

We studied the CME that had a complex acceleration profile: two impulsive acceleration phases and one impulsive deceleration phase. The CME showed signatures of several acceleration mechanisms: kink instability, mass drainage, flare reconnection, and CME-CME collision.

The kink instability triggered the CME eruption. The prominence drainage happened after the kink instability.
The drainage played an auxiliary role: it decreased the CME mass, which helped its acceleration.

The first impulsive acceleration phase was caused by the flare reconnection below the CME. During the first phase, the two-ribbon flare occurred and the SphinX Xray flux increased.

The second impulsive acceleration and a deceleration phase were caused by the CME-CME interaction. We think that the deceleration corresponds to CME compression, and the acceleration to CME restitution.

The studied event shows that CMEs are complex phenomena that cannot be explained by only one acceleration mechanism. We should seek a combination of different mechanisms that accelerate CMEs at different stages of their evolution.

This research was supported by the Russian Science Foundation (project No. 17-12-01567).

\section{REFERENCES}

Akmal, A., Raymond, J. C., Vourlidas, A., et al. 2001, ApJ, 553, 922

Alissandrakis, C. E., Kochanov, A. A., Patsourakos, S., et al. 2013, PASJ, 65, 8

Antiochos, S. K., DeVore, C. R., \& Klimchuk, J. A. 1999, ApJ, 510,485
Aulanier, G. 2014, in IAU Symposium, Vol. 300, Nature of Prominences and their Role in Space Weather, ed. B. Schmieder, J.-M. Malherbe, \& S. T. Wu, 184-196

Bein, B. M., Berkebile-Stoiser, S., Veronig, A. M., Temmer, M., \& Vršnak, B. 2012, ApJ, 755, 44

Bemporad, A., Raymond, J., Poletto, G., \& Romoli, M. 2007, ApJ, 655, 576 

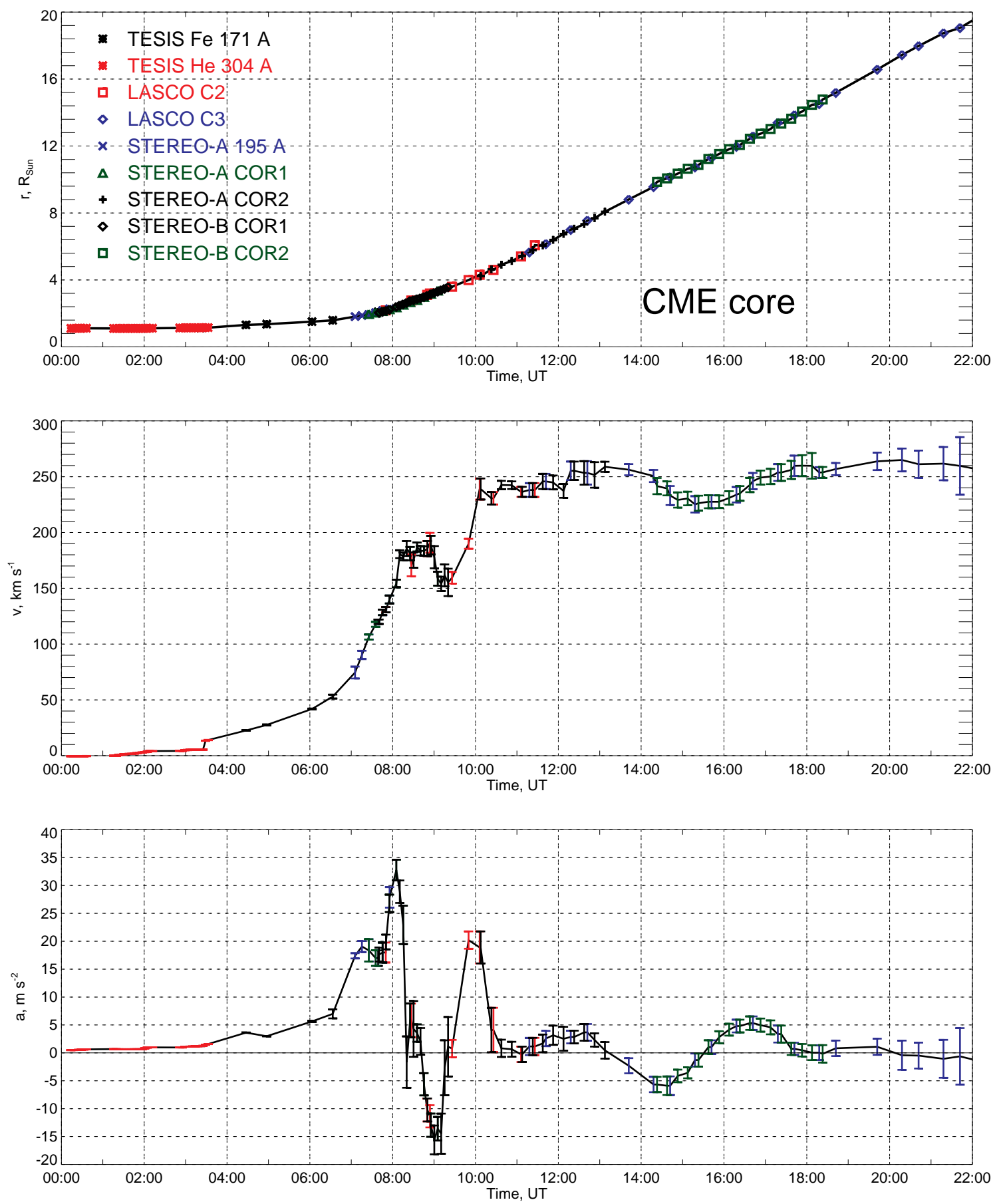

Figure 11. The kinematics of the CME core. $r(t)$ is the distance from the CME core to the Sun's center, $v(t)$ is the CME core radial velocity, and $a(t)$ is the CME core radial acceleration.

Brueckner, G. E., Howard, R. A., Koomen, M. J., et al. 1995, SoPh, 162, 357

Byrne, J. P., Morgan, H., Seaton, D. B., Bain, H. M., \& Habbal, S. R. 2014, SoPh, 289, 4545

Carmichael, H. 1964, NASA Special Publication, 50, 451

Chen, P. F. 2011, Living Reviews in Solar Physics, 8, doi:10.12942/lrsp-2011-1

Ciaravella, A., Raymond, J. C., van Ballegooijen, A., et al. 2003, ApJ, 597, 1118
Ciaravella, A., Raymond, J. C., Fineschi, S., et al. 1997, ApJL, 491, L59

Ciaravella, A., Raymond, J. C., Strachan, L., et al. 1999, ApJ, 510, 1053

Ciaravella, A., Raymond, J. C., Thompson, B. J., et al. 2000, ApJ, 529, 575

Delaboudinière, J., Artzner, G. E., Brunaud, J., et al. 1995, SoPh, 162, 291 

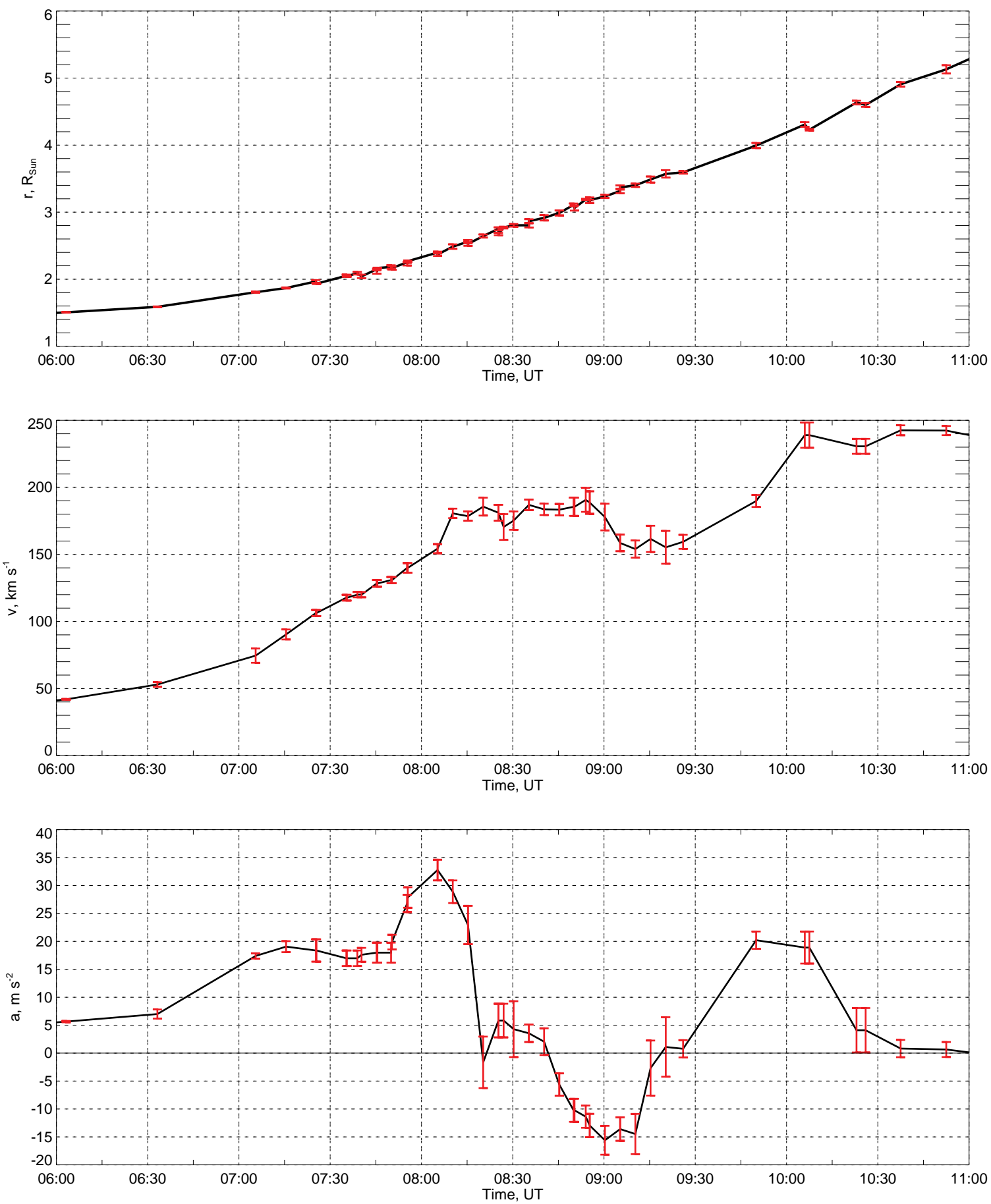

Figure 12. The kinematics of the CME core. Zoomed version of Figure 11. $r(t)$ is the distance from the CME core to the Sun's center, $v(t)$ is the CME core radial velocity, and $a(t)$ is the CME core radial acceleration.

D'Huys, E., Seaton, D. B., De Groof, A., Berghmans, D., \&

Poedts, S. 2017, Journal of Space Weather and Space Climate,

7, A7

Fan, Y., \& Low, B. C. 2003, in Astronomical Society of the Pacific Conference Series, Vol. 286, Current Theoretical Models and Future High Resolution Solar Observations:

Preparing for ATST, ed. A. A. Pevtsov \& H. Uitenbroek, 347

Filippov, B., \& Koutchmy, S. 2002, SoPh, 208, 283
Gburek, S., Sylwester, J., Kowalinski, M., et al. 2011, Solar System Research, 45, 189

Gosain, S., Filippov, B., Ajor Maurya, R., \& Chandra, R. 2016 , ApJ, 821, 85

Hirayama, T. 1974, SoPh, 34, 323

Hood, A. W., \& Priest, E. R. 1981, Geophysical and Astrophysical Fluid Dynamics, 17, 297

House, L. L., Wagner, W. J., Hildner, E., Sawyer, C., \& Schmidt, H. U. 1981, ApJL, 244, L117 

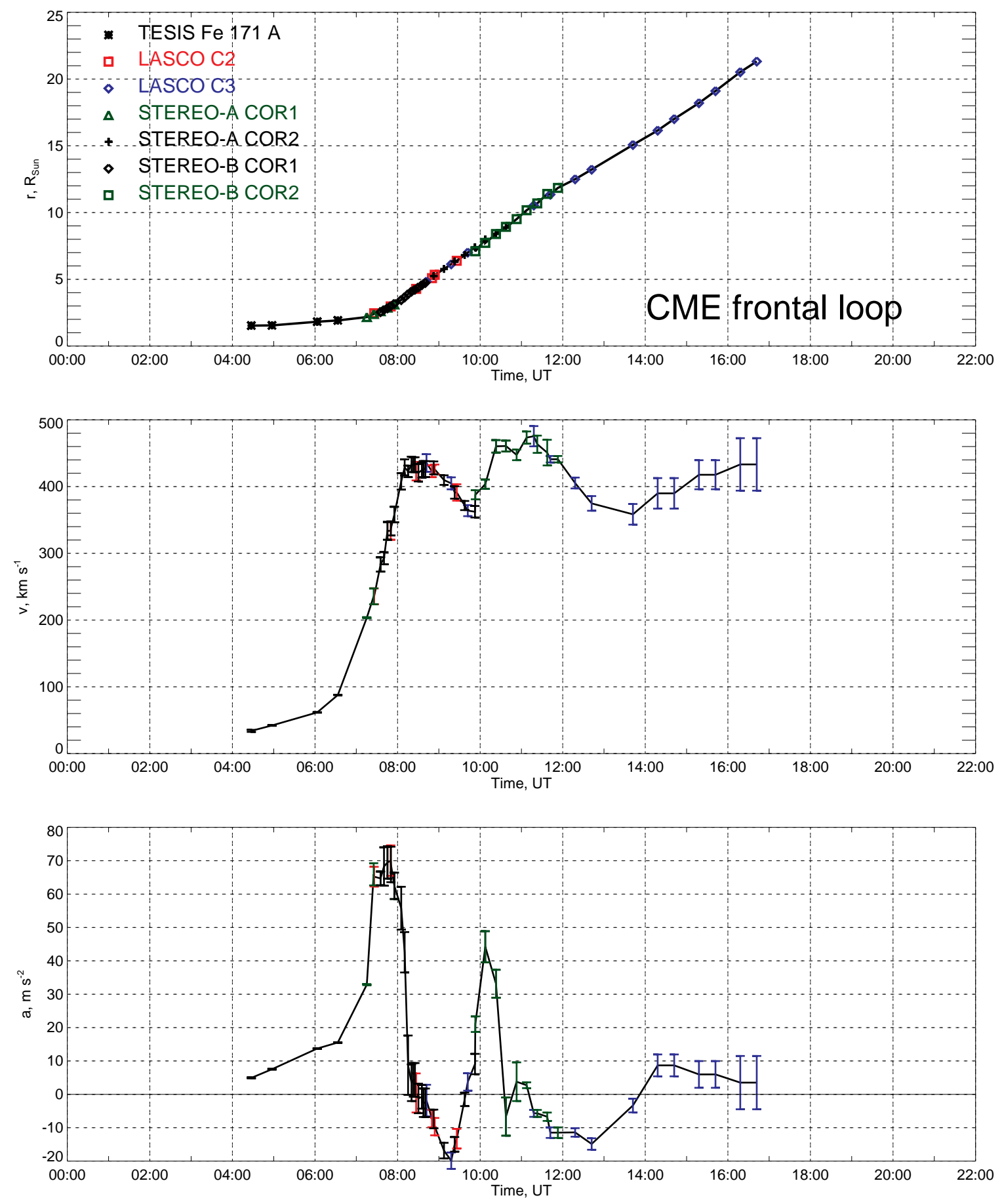

Figure 13. Kinematics of the CME frontal loop. $r(t)$ is the distance from the CME frontal loop to the Sun's center, $v(t)$ is the CME frontal loop radial velocity, and $a(t)$ is the CME frontal loop radial acceleration.

Howard, R. A., Moses, J. D., Vourlidas, A., et al. 2008, SSRv, 136,67

Hundhausen, A. 1999, in The many faces of the sun: a summary of the results from NASA's Solar Maximum Mission., ed. K. T. Strong, J. L. R. Saba, B. M. Haisch, \& J. T. Schmelz, 143 Illing, R. M. E., \& Athay, G. 1986, SoPh, 105, 173

Illing, R. M. E., \& Hundhausen, A. J. 1985, J. Geophys. Res., 90,275

Kirichenko, A. S., \& Bogachev, S. A. 2017a, ApJ, 840, 45
一. 2017b, SoPh, 292, 120

Kliem, B., \& Török, T. 2006, Physical Review Letters, 96, 255002 Kopp, R. A., \& Pneuman, G. W. 1976, SoPh, 50, 85

Kotov, Y. D. 2011, Solar System Research, 45, 93

Kuzin, S. V., Zhitnik, I. A., Shestov, S. V., et al. 2011, Solar System Research, 45, 162

Landi, E., Raymond, J. C., Miralles, M. P., \& Hara, H. 2010, ApJ, 711, 75

Lemen, J. R., Title, A. M., Akin, D. J., et al. 2011, SoPh, 172 

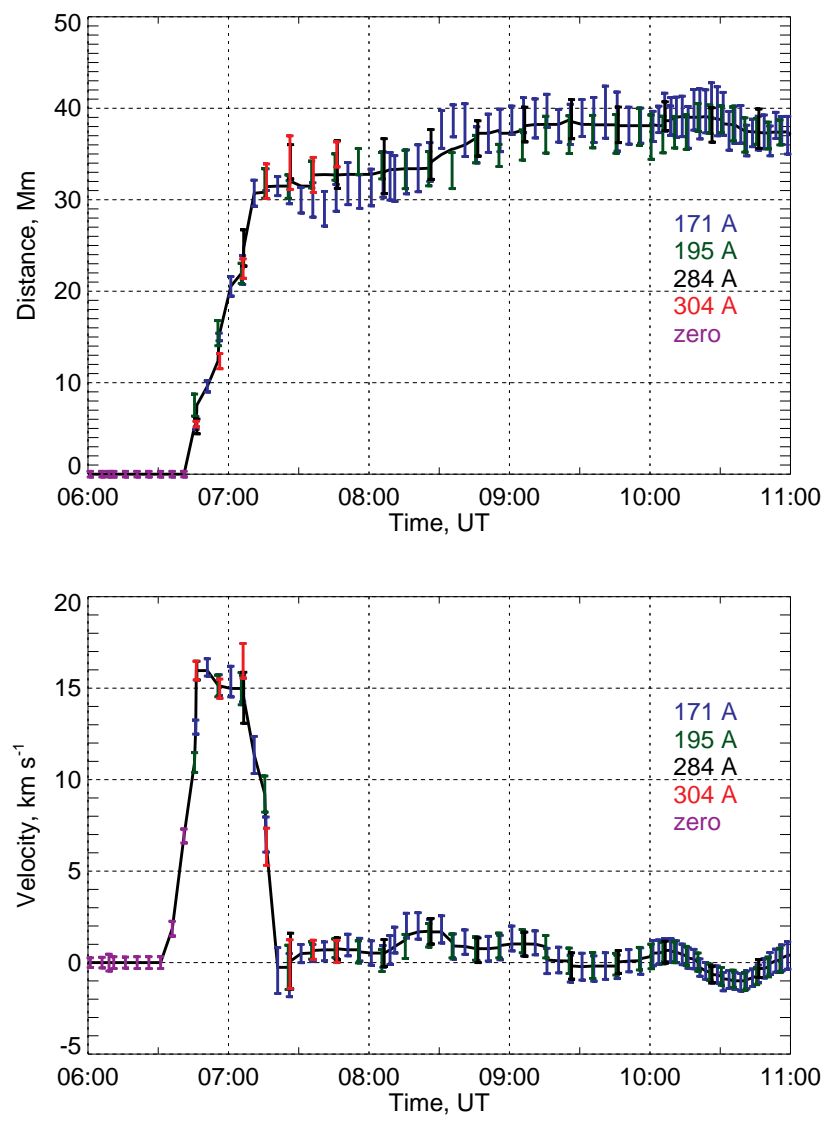

Figure 14. The distance between the flare ribbons (top) and the velocity of the ribbon separation (bottom). Blue: EUVIB $171 \AA$ channel; green: EUVI-B $195 \AA$ channel; black: EUVI-B $284 \AA$ A channel; red: EUVI-B $304 \AA$ channel; purple: "zero points" (171 A images without flare ribbons).

Lugaz, N., Temmer, M., Wang, Y., \& Farrugia, C. J. 2017, SoPh, 292, 64

Maričić, D., Vršnak, B., Stanger, A. L., et al. 2007, SoPh, 241, 99

Mierla, M., Seaton, D. B., Berghmans, D., et al. 2013, SoPh, 286,241

Neupert, W. M. 1968, ApJL, 153, L59
Nindos, A., Patsourakos, S., Vourlidas, A., \& Tagikas, C. 2015, ApJ, 808, 117

Parenti, S. 2014, Living Reviews in Solar Physics, 11, doi:10.1007/lrsp-2014-1

Plunkett, S. P., Vourlidas, A., Šimberová, S., et al. 2000, SoPh, 194, 371

Qiu, J., Wang, H., Cheng, C. Z., \& Gary, D. E. 2004, ApJ, 604, 900

Reeves, K. K., \& Forbes, T. G. 2005, in IAU Symposium, Vol. 226, Coronal and Stellar Mass Ejections, ed. K. Dere, J. Wang, \& Y. Yan, 250-255

Reeves, K. K., \& Golub, L. 2011, ApJL, 727, L52

Reva, A. A., Ulyanov, A. S., Bogachev, S. A., \& Kuzin, S. V. 2014, ApJ, 793, 140

Reva, A. A., Ulyanov, A. S., \& Kuzin, S. V. 2016a, ApJ, 832, 16

Reva, A. A., Ulyanov, A. S., Shestov, S. V., \& Kuzin, S. V. 2016b, ApJ, 816, 90

Schmieder, B., Aulanier, G., \& Vršnak, B. 2015, SoPh, 290, 3457

Seaton, D. B., Mierla, M., Berghmans, D., Zhukov, A. N., \& Dolla, L. 2011, ApJL, 727, L10

Shen, C., Wang, Y., Wang, S., et al. 2012a, Nature Physics, 8, 923

Shen, F., Shen, C., Wang, Y., Feng, X., \& Xiang, C. 2013, Geophys. Res. Lett., 40, 1457

Shen, F., Wu, S. T., Feng, X., \& Wu, C.-C. 2012b, Journal of Geophysical Research (Space Physics), 117, A11101

Slemzin, V., Bougaenko, O., Ignatiev, A., et al. 2008, Annales Geophysicae, 26, 3007

Song, H. Q., Zhang, J., Chen, Y., \& Cheng, X. 2014, ApJL, 792, L40

Sturrock, P. A. 1966, Nature, 211, 695

Su, Y., Dennis, B. R., Holman, G. D., et al. 2012, ApJL, 746, L5

Temmer, M., Veronig, A. M., Kontar, E. P., Krucker, S., \& Vršnak, B. 2010, ApJ, 712, 1410

Temmer, M., Veronig, A. M., Vršnak, B., et al. 2008, ApJL, 673, L95

Török, T., \& Kliem, B. 2005, ApJL, 630, L97

Webb, D. F., \& Howard, T. A. 2012, Living Reviews in Solar Physics, 9, doi:10.12942/lrsp-2012-3

Webb, D. F., \& Hundhausen, A. J. 1987, SoPh, 108, 383

Wood, G. A. 1982, Exercise and Sport Sciences Reviews, 10, 308

Yashiro, S., Gopalswamy, N., Michalek, G., et al. 2004, Journal of Geophysical Research (Space Physics), 109, 7105

Zhang, J., Dere, K. P., Howard, R. A., Kundu, M. R., \& White, S. M. 2001, ApJ, 559, 452 